\title{
EM Telemetry \\ Tool for Deep \\ Well Drilling \\ Applications
}

\section{Final Report}

\author{
Prepared by \\ Jeffrey M. Gabelmann P.E \\ Prepared November 15, 2005. \\ DOE Award Number DE-FC26-02NT41656
}

\author{
E-Spectrum Technologies, Incorporated \\ 12725 Spectrum Drive, San Antonio, Texas 78249 \\ www.espectech.com
}




\section{DISCLAIMER}

"This report was prepared as an account of work sponsored by an agency of the United States Government. Neither the United States Government nor any agency thereof, nor any of their employees, makes any warranty, express or implied, or assumes any legal liability or responsibility for the accuracy, completeness, or usefulness of any information, apparatus, product, or process disclosed, or represents that its use would not infringe privately owned rights. Reference herein to any specific commercial product, process, or service by trade name, trademark, manufacturer, or otherwise does not necessarily constitute or imply its endorsement, recommendation, or favoring by the United States Government or any agency thereof. The views and opinions of authors expressed herein do not necessarily state or reflect those of the United States Government or any agency thereof." 


\begin{abstract}
This final report discusses the successful development and testing of a deep operational electromagnetic (EM) telemetry system, produced under a cooperative agreement with the United States Department of Energy's National Energy Technology Laboratory. This new electromagnetic telemetry system provides a wireless communication link between sensors deployed deep within oil and gas wells and data acquisition equipment located on the earth's surface. EM based wireless telemetry is a highly appropriate technology for oil and gas exploration in that it avoids the need for thousands of feet of wired connections. In order to achieve the project performance objectives, significant improvements over existing EM telemetry systems were made. These improvements included the development of new technologies that have improved the reliability of the communications link while extending operational depth. A key element of the new design is the incorporation of a data-fusion methodology which enhances the communication receiver's ability to extract very weak signals from large amounts of ambient environmental noise. This innovative data-fusion receiver based system adapts advanced technologies, not normally associated with low-frequency communications, and makes them work within the harsh drilling environments associated with the energy exploration market. Every element of a traditional EM telemetry system design, from power efficiency to reliability, has been addressed. The data fusion based EM telemetry system developed during this project is anticipated to provide an EM tool capability that will impact both onshore and offshore oil and gas exploration operations, for conventional and underbalanced drilling applications.
\end{abstract}




\section{TABLE OF CONTENTS}

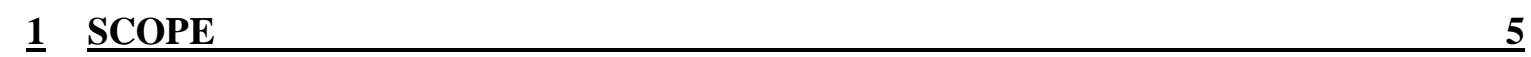

$\underline{2}$ PROJECT GOAL

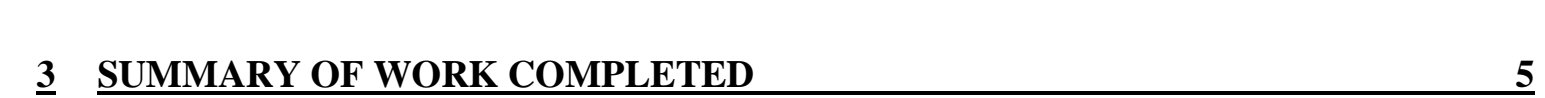

\begin{tabular}{|l|r|r|}
\hline & & \\
\hline \hline 3.1 & DATA FUSION RECEIVER DEVELOPMENT & $\mathbf{5}$ \\
\hline 3.1 .1 & DATA FUSION ALGORITHM DEVELOPMENT & 5 \\
\hline 3.1 .2 & DATA FUSION RECEIVER HARDWARE PLATFORM DEVELOPMENT & 5 \\
\hline $\mathbf{3 . 2} \quad$ HIGH EFFICIENCY POWER AMPLIFIER DEVELOPMENT & $\mathbf{7}$ \\
\hline \hline 3.2 .1 & DOWNHOLE TOOL POWER AMPLIFIER AND RECEIVER & 9 \\
\hline 3.2 .2 & SURFACE POWER AMPLIFIER & $\mathbf{1 0}$ \\
\hline $\mathbf{3 . 3} \quad$ FIELD TESTING & 10 \\
\hline 3.3 .1 & TEST-WELL PERFORMANCE TESTING & 11 \\
\hline 3.3 .2 & FIELD NOISE RECORDINGS & \\
\hline
\end{tabular}

4 RESULTS AND DISCUSSION

$4.1 \quad$ FieLd Test Results

\begin{tabular}{|lrl}
\hline 4.1 .1 & TEST-WELL PERFORMANCE TESTING RESULTS & 12 \\
\hline
\end{tabular}

\begin{tabular}{|lll}
\hline 4.1 .2 & FIELD NOISE RECORDING TEST RESULTS & 13 \\
\hline
\end{tabular}

\begin{tabular}{|lr|}
\hline 4.2 & INTELLECTUAL PROPERTY DEVELOPMENT \\
\hline
\end{tabular}

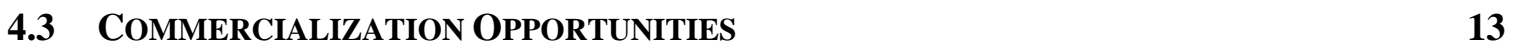

\begin{tabular}{|lrr}
\hline \hline .3 .1 & POTENTIAL ALTERNATIVE MARKET AREAS & 14 \\
\hline
\end{tabular}

\begin{tabular}{|ll|}
\hline CONCLUSIONS & 16 \\
\hline
\end{tabular} 


\section{Scope}

This document constitutes the final report for DOE project award number DE-FC2602NT41656 entitled "EM Telemetry for Deep Drilling Applications".

\section{Project Goal}

The goal of the project was to design and develop a commercially viable subterranean wireless communication system capable of prolonged operation at extended vertical deployment depths.

\section{Summary of Work Completed}

The work completed during the project performance period included the development of a working prototype of a Data Fusion Receiver and High Efficiency Power Amplifier. Project work also included field testing of the prototypes. The following sections describe each type of work product in detail.

\subsection{Data Fusion Receiver Development}

The development of the data fusion receiver consisted of two distinct work areas: the development and tuning of the data fusion algorithms and the design and construction of a data fusion receiver hardware platform. The following sections describe each work effort.

\subsubsection{Data Fusion Algorithm Development}

The objective of the data fusion algorithm development work was to develop and validate a set of innovative signal processing algorithms designed to maximize the noise rejection capabilities of a high-sensitivity subterranean communications receiver. Increasing a receiver's noise rejection capability provides the receiver with the ability to recover and demodulate very small amplitude signals in the presence of large amounts of interfering ambient noise. Within the context of the Deep Trek project objectives, developing a methodology which increases a subterranean telemetry receiver's noise rejection capability improves the signal-to-noise ratio parametric of said receiver, thus making the receiver capable of recovering the extremely weak electromagnetic field signals associated with deep subterranean telemetry applications.

\subsubsection{Data Fusion Receiver Hardware Platform Development}

The objective of the data fusion receiver hardware platform development work was to develop a novel new ultra-low frequency electromagnetic telemetry receiver apparatus that utilizes data fusion methods to fuse multiple input-receive sources into a single decodable message packet. Further more, it was an objective that the data fusion receiver platform be made rugged and compact in order to satisfy the requirements of the targeted commercial market; specifically, real-time subterranean telemetry for the oil and gas exploration industry. 
The receiver platform developed during the Deep Trek project consists of two primary components: an ultra low-noise signal conditioning box and a Windows-based computer. Figure 1 is a photograph of the Data Fusion Receiver platform developed during the project.

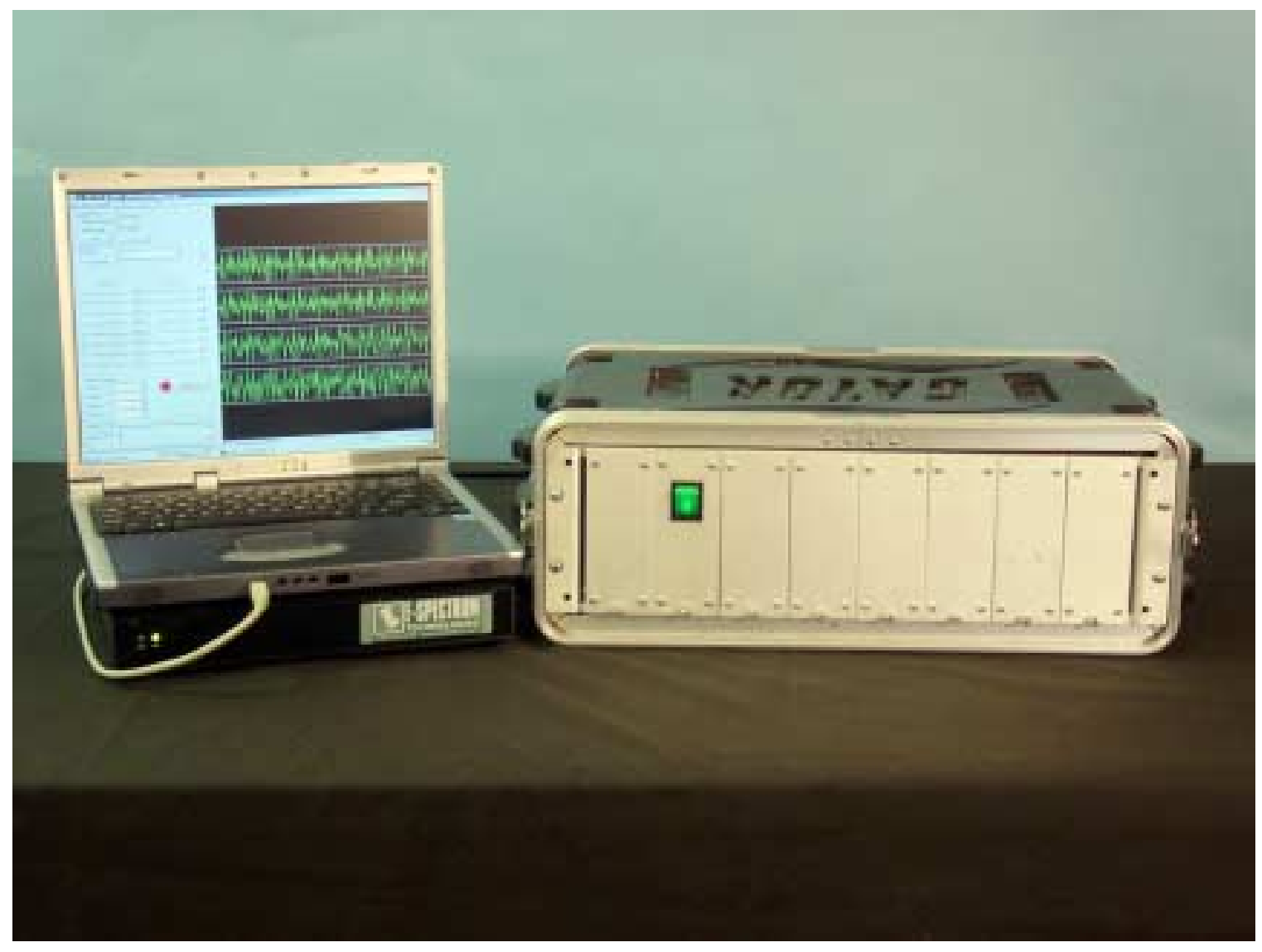

Figure 1. Data Fusion Receiver Hardware Platform.

The ultra low noise signal-conditioning box (right-hand side of Figure 1) provides signal conditioning for up to four electric-field signal channels and up to twelve magnetic-field signal channels; that is, a total of sixteen real-time receive-input channels.

The signal-conditioning box provides the following capabilities:

- Sixteen, Zero-phase, fourth-order band-pass filters $(1 \mathrm{~Hz}$ to $20 \mathrm{~Hz})$

- $84 \mathrm{~dB}$ of adjustable gain (in 6dB increments)

- 850nVRMS sensitivity

The low-noise signal-conditioning electronics are housed in a rugged, compact enclosure (20"x 8"x16" - WxHxD) which provides MIL-SPEC field-wiring connections for the external electric and magnetic field antennas. 
A Windows-based, $\mathrm{PC}$-architecture computer (the laptop computer shown in the left portion of Figure 1) provides an industry standard platform for digitizing the incoming signal channels and also provides the run-time processing platform for the data fusion and demodulation engines. The PC also hosts the data fusion receiver's graphical user interface (GUI) application. The primary function of the GUI is to provide the operatorinterface for display of the data received from the downhole tool. This data can be realtime information from the tool sensors (e.g. tool-face information for MWD applications) or diagnostic information about the "health" of the tool (e.g. battery voltages, gap antenna impedance, etc.). The GUI application also allows the operator to format downlink commands to the downhole tool in order to configure the tool for telemetry uplinks or to query the tool for real-time health/diagnostic information. Additional GUI functionality includes the ability to display multiple incoming receive antenna waveforms, allowing the operator to select any four of the sixteen incoming waveforms for real-time display. The data fusion receiver application also allows the user to save the incoming real-time waveform data to the $\mathrm{PC}$ hard-drive to facilitate post-processing analysis of the raw temporal-waveform data. In addition, the data fusion receiver application keeps an electronic log of all uplinks received from the tool and all downlinks sent to the tool. This log is time stamped and can be used to facilitate the post-processing of the raw waveform data archived to the hard-drive.

The usage of the industry standard Windows-based, PC-architecture operating platform provides the data fusion receiver system greater operational flexibility and helps to mitigate obsolescence of the hardware components. Operational flexibility is engendered by the hosting of the data fusion and receive engine applications over the standardized Windows XP operating system. This allows both processing engines to be easily upgraded with regards to processing power and algorithmic capability. Upgrades are facilitated by simply porting the applications to a higher performance laptop or desktop PC. Hardware obsolescence is mitigated via the standardized PC-architecture platform which is universally supported by thousands of plug-in peripheral component manufacturers.

\subsection{High Efficiency Power Amplifier Development}

The objective of the high efficiency power amplifier (PA) development work was to develop a novel new communications transmitter PA architecture which provides increased power-delivery efficiency in a compact, rugged mechanical package. Increasing transmission efficiency results in a greater transfer of available energy from the transmitter's principal power source to the transmission media. The maximization of energy transfer is especially critical downhole, where the transmitter's principal power source is typically a battery. Efficient coupling of magnetic field energy into the low impedance transmission media present at the PA transmitting antenna results in a maximum amount of field energy remaining at the receiver antenna after transit through the earth's strata. Increased field energy at the receive antenna equates to increased recoverable signal amplitude; thus, the overall receiver signal-to-noise ratio is improved resulting in increased operational depth capability. 
Two separate high efficiency power amplifier systems were developed during the project: a downhole tool power amplifier and a surface power amplifier. The following sections describe the development of each power amplifier system.

\subsubsection{Downhole Tool Power Amplifier and Receiver}

The objective of the downhole tool power amplifier work was to develop a compact and rugged PA which utilizes a solid-state switched-output driver topology to efficiently convert stored energy from a battery into magnetic field energy. For maximum operational flexibility, the downhole PA must be capable of re-configuration while deployed in-hole; thus, it was a requirement that the PA have receive capability to facilitate a two-way, EM-based, communications link to the surface. In addition, it was an objective that the downhole tool PA electronic-assembly be sized such that it would fit within a 1.25 " I.D. (max) pressure vessel to allow for integration into a working drillstring.

Figure 2 is a photograph of the downhole tool power amplifier and receiver assembly developed during the project. The tool assembly consists of two printed circuit boards mounted in a rigid backbone. Each circuit board is designed to utilize SOIC components provided by Honeywell. The Honeywell SOIC components are designed for extended temperature range operation to $225^{\circ} \mathrm{C}$ and are provided in thru-hole style integrated circuit packages.

The power source for the downhole electronic assembly consists of a battery pack constructed from a series of high-temperature $\left(200^{\circ} \mathrm{C}\right)$, lithium-thionyl-chloride batteries arranged in an axial configuration (shown in the right-hand portion of Figure 2). The battery pack is assembled with insulators between each cell to prevent shorting during drilling operations and to act as mini shock absorbers to absorb vibration. The entire $\mathrm{PA} /$ receiver/battery assembly is contained within a 1.25" I.D. screw-on pressure housing (shown in the foreground of the photograph).

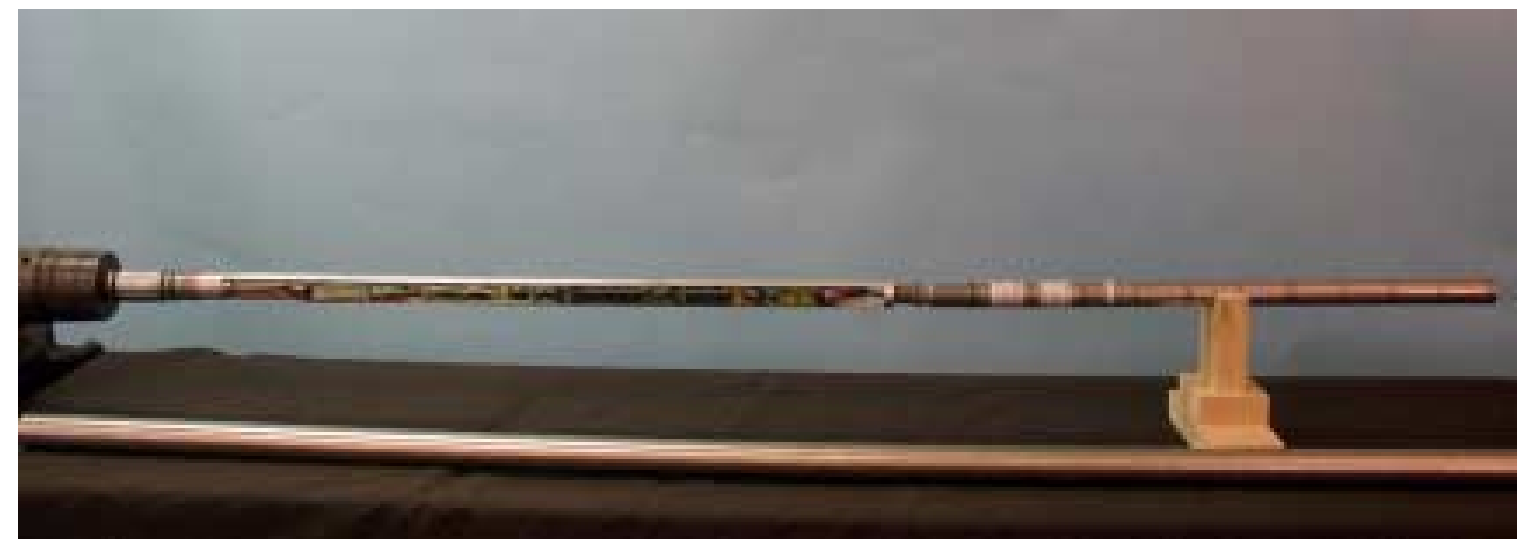

Figure 2. Downhole Tool Power Amplifier and Receiver Assembly. 
The downhole tool PA/receiver assembly provides the following capabilities:

- $15 \mathrm{Wrms}$ (max) delivered power: programmable via the EM downlink (8 adjustment settings).

- $2 \mathrm{~Hz}$ to $10 \mathrm{~Hz}$ adjustable uplink carrier frequency: programmable via the EM downlink (five adjustment settings).

- Ability to measure received signal strength of the downlink and report via EM uplink.

- Ability to measure gap-antenna impedance and report via EM uplink.

- Ability to perform real-time system-health diagnostics and report status via EM uplink.

- Ability to record up to 30 minutes of time domain waveforms from the gap antenna.

\subsubsection{Surface Power Amplifier}

The objective of the Surface Power Amplifier work was to develop a compact and rugged power amplifier to facilitate downlink command-communications with the downhole tool. Figure 3 is a photograph of the Surface Power Amplifier developed during the project.

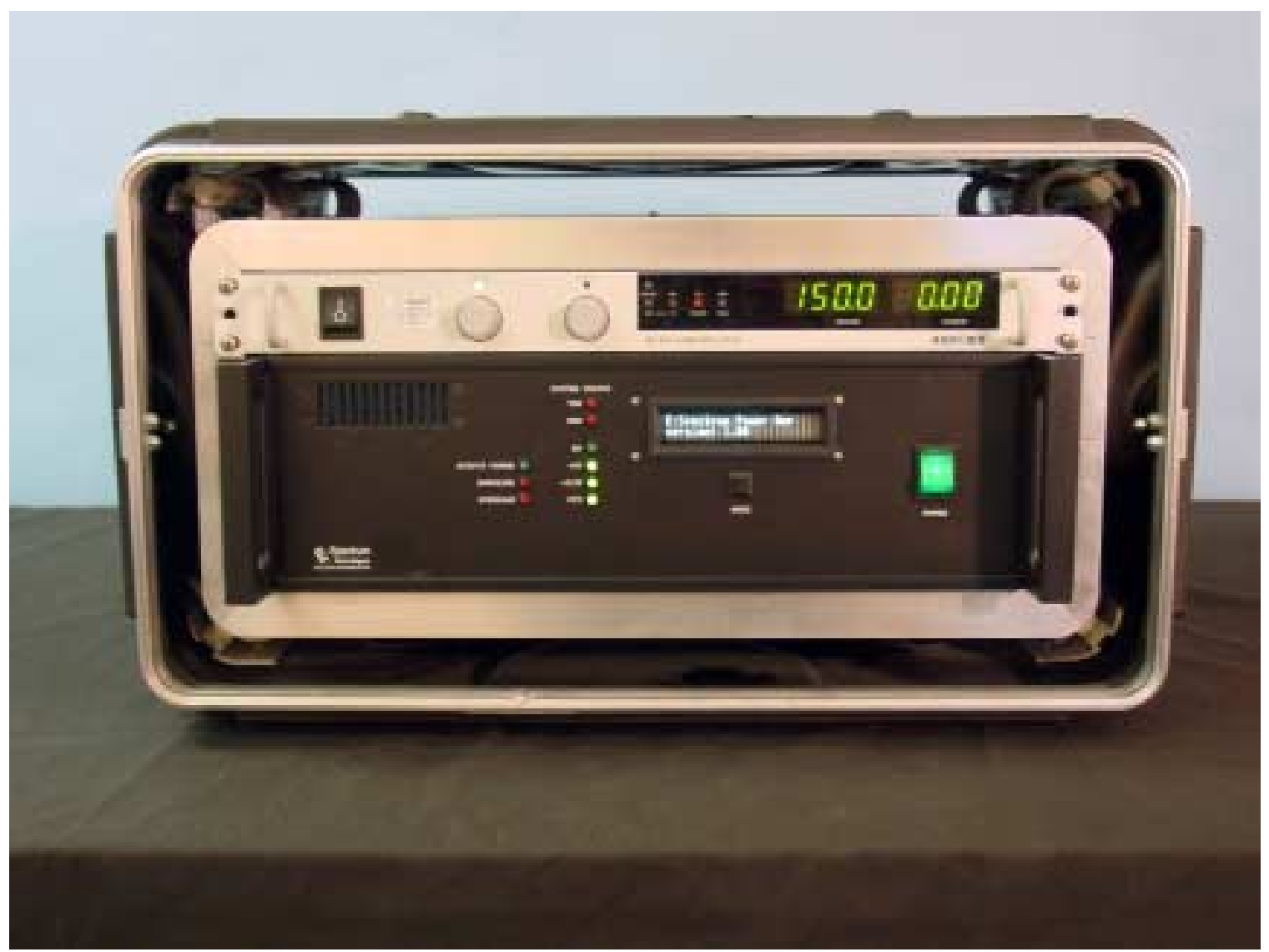

Figure 3. Surface Power Amplifier. 
The Surface Power Amplifier electronics are contained in a rugged 19", $8 \mathrm{U}$ rack. The Surface power amplifier electronics utilize the same basic solid-state, switched output driver topology as the downhole tool PA; however, the Surface PA is capable of generating $1000 \mathrm{Wrms}$ of power to the load. In addition, the surface PA features safety interlocks whereby power delivery to the load is discontinued or disabled when an abnormal impedance condition, such as an open-circuit, is sensed on the PA outputs. This interlock feature helps to protect against the safety hazard caused by broken, loose, or improperly connected antenna wires.

The Surface PA provides an RS-232 serial communications interface to a laptop or desktop computer whereby information can be exchanged with the data fusion receiver GUI application. The serial communications link is used to relay the operator-downlink command packet to the PA for formatting and subsequent downlink transmission to the downhole tool. The Surface PA also performs internal health/diagnostic functions such as measurement of the transmission antenna load impedance and the monitoring of various internal system-health metrics. This data is reported to the data fusion receiver GUI application via the RS-232 serial communications interface.

\subsection{Field Testing}

A total of seven separate field tests were conducted during the project to facilitate the development, tuning, and validation of the Deep Trek data fusion system. Field testing consisted of two basic types of test protocols: Test-well performance testing, and fieldnoise recordings. The following sections describe the test setup and protocol for each type of field test.

\subsubsection{Test-well Performance Testing}

The test-well performance tests were conducted in two commercial test well facilities. Facility number 1 consisted of a 5000' deep cased hole ( $71 / 2$ " casing at 5000') topped by a fully equipped drilling-rig platform. Facility number 1 was ideal for simulation of deep operational deployments within an uncased hole due to the shielding attenuation provided by the relatively narrow casing.

Three separate series of tests were performed at Facility Number 1. These tests occurred on the following dates:

- February $2004-3$ days

- May of $2004-2$ days

- August of $2004-2$ days

Facility Number 2 consisted of a 1000' deep cased hole (30" casing at 1000') topped by a fully equipped drilling-rig platform. Facility Number 2 was ideally suited for 
simulating low-resitivity formation, deep, open-hole operation because of the large diameter of the casing.

One set of tests was performed at Facility Number 2. This test occurred on the following date:

- June 2005 - 1 day

All of the test-well performance tests utilized the same basic protocol. This protocol is as follows:

- Run-tool into well to the desired operational depth.

- Command tool to send $\boldsymbol{x}$ number of uplinks (referred to as a batch) at $\boldsymbol{y}$ output power and $z$ carrier frequency.

- Begin rotating the drill-string/tool after the beginning of the first uplink is detected.

- Determine how many uplinks within the batch were decodable.

- Lower the tool output power and repeat test until no uplinks within the batch are decodable.

Appendix A contains a sample test-well performance test protocol.

\subsubsection{Field Noise Recordings}

Field-noise recordings were conducted on working drilling rigs at various locations throughout Texas. The recordings were made utilizing the Data Fusion Receiver's ability to record raw waveforms direct to hard-disk. The basic test protocol consisted of deploying several electric and magnetic field antennas around a working drilling rig to record the electric and magnetic field noise generated by the rig. Recorded data was analyzed for harmonic content and the results were compared to rig-noise generated during the test well performance tests to validate that the noise encountered in an actual field environment is similar to the noise used to test/tune the data fusion algorithms. Three separate field wells were used for field noise recordings. 


\section{Results and Discussion}

\subsection{Field Test Results}

The following sections provide a brief description of the results of the two types of field testing conducted during the project. Refer to the cited appendices for specific test result details.

\subsubsection{Test-well Performance Testing Results}

Four separate test-well performance tests were conducted. The following is a brief synopsis of each test. Detailed test results for each test are located in the indicated Appendices of this document.

February 2004 - Facility Number 1

This test consisted of data recording and, as such, has no directly related performance metric to report. Three days worth of rotational noise data was gathered and archived for use in algorithm development and tuning.

\section{May 2004 - Facility Number 1}

This test was the initial test-deployment of the real-time data fusion algorithms using the data fusion receiver hardware test platform and downhole tool. Testing at a depth of 2200' resulted in 27 of 30 transmissions being recovered in the presence of strong rotational noise yielding a 90\% recovery rate. Testing at a depth of 4944' resulted in 26 of 61 transmissions being recovered in the presence of strong rotational noise yielding a $43 \%$ recovery rate.

August 2004 - Facility Number 1

This test was conducted to verify that the tuning upgrades made to the real-time data fusion algorithms, based on the post-analysis of data gathered during the May 2004 testing, were effective. Test data recorded during the May 2005 test was used to tune the data fusion algorithms for optimal performance. After the improved algorithms were added to the data fusion receiver hardware platform, the hardware test platform and downhole tool were deployed at the test well for two days of testing. Testing at a depth of 4944' resulted in 68 of 80 transmissions being recovered in the presence of strong rotational noise giving an $85 \%$ recovery rate. It should be noted that these transmissions were recovered while the downhole tool was set at the lowest possible power rating (less than $1.7 \mathrm{~W}$ ) making this test an extremely successful demonstration of the data fusion receivers ability to recover extremely weak signal in the presence of strong rotational noise. Post analysis and subsequent tuning and reprocessing of the raw data waveforms resulted in an additional seven uplinks being recovered yielding a final recovery rate of $94 \%$.

June 2005 - Facility Number 2

This test was conducted to simulate the operation of the data fusion system in an open (i.e. uncased) hole. Facility Number 2 is constructed using 30" casing down to 1000'. When filled with a conductive fluid (e.g. salt water), a well with large diameter casing 
can provide a good simulation of deep deployment in an open-hole formation. Mathematical modeling of the Facility Number 2 test well, based on casing thickness, transmission power, and fluid conductivity predicts that operation at 1000' in the existing casing configuration is equivalent to 11,000 ' to 13,000 ' operation in a 7' bore open-hole (dependant on transmission power). Testing at a depth of 1000' resulted in 52 of 68 transmissions being recovered in the presence of strong rotational noise yielding a $77 \%$ recovery rate.

\subsubsection{Field Noise Recording Test Results}

Field noise recording test results showed that the harmonic content of a variety of actual drilling rigs was similar to that encountered in both test-wells. It was found that the severity of the noise encountered at the working drilling rigs was actually much less (based on average amplitude) than that encountered in the test wells. This would indicate that worst case noise conditions were used to develop and tune the data fusion algorithms.

\subsection{Intellectual Property Development}

A patent has been applied for regarding the Data-fusion Receiver apparatus and algorithms. The expected award date is February 2006.

\subsection{Commercialization Opportunities}

One of the stated goals of this project was to develop an enabling technology that has widespread commercial viability; that is, a technology that has the ability to favorably impact a specific market and generate revenue. Field test results indicate that the data fusion technology provides a measurable advantage over existing EM systems and thus will have a favorable impact on the commercial market.

It is envisioned that the data fusion technology will be introduced into the energy exploration market via a partnership arrangement between E-Spectrum Technologies and an industry partner. Potential industry partners within the identified target market include: small, independent MWD service providers; large energy services companies; and large energy/oil companies. A brief description of each type of potential partnership arrangement is presented in the following sections.

\section{Small MWD Service Providers}

This type of potential commercial partner consists of smaller companies that, typically, have less access to capital and development resources than the larger full-service providers. A small service provider might not have access to any type of EM telemetry technology and might primarily rely on mud-pulse or wireline telemetry systems to facilitate their MWD services. A business arrangement with such a partner would most likely involve a leasing or per-use type of revenue generation model. 


\section{Large Energy Services Companies}

This type of service provider would typically have a large operating budget and would most likely have access to some type of EM telemetry system: either developed in-house or purchased from a third party. A business arrangement with this type of partner would most likely involve providing key enabling technologies, via a licensing agreement, to augment their existing EM MWD capabilities.

\section{Energy/oil Companies}

A partnership arrangement with a large energy/oil company would most likely involve seeking direct $\mathrm{R} \& \mathrm{D}$ funding geared towards a more general investment in developing technologies for use across multiple sectors in the drilling industry. This approach would entail the acquisition of additional development funding to expand the applicability of the basic data fusion technique to other technology sectors within the energy industry.

\subsubsection{Potential Alternative Market Areas}

Energy exploration applications are considered the primary market area for the data fusion technologies developed under this project; however, certain key subsystems might have applications within alternative market areas. These key marketable subsystems include: the data fusion signal processing algorithms, the high-efficiency power amplifier topology and techniques, and the down-hole electronics package. In aggregate, these technological elements combine to create the major components of a complete EM telemetry system. However, it is feasible that subcomponents of this electronics/software suite can be used to enhance the operational capabilities of other types of telemetry or information gathering systems by addressing key limiting factors such as dynamic noise rejection, low noise floor, efficient power delivery, etc. Potential alternative markets include the following application areas:

\section{Seabed Logging EM}

Seismic imaging techniques can detect subsurface hydrocarbons by an active source electromagnetic (EM) sounding application, termed seabed logging (SBL). This technology has recently shown promising results. Recently, it has been demonstrated that this measurement technique may reliably provide direct detection of a deeply buried hydrocarbon accumulation by electromagnetic sounding. Use of the data fusion engine developed under this effort could further enhance the efficacy of this type of system in commercial applications.

\section{Robust Emergency Mine Communications}

Emergency Mine Communications systems for underground coalmines to enable bidirectional, low bit-rate data communications between the surface control room and personnel underground have recently been developed. Wireless EM equipment does not rely on other communication systems and mine infrastructure to remain intact after a disaster. Several elements of the innovations resulting from this research can be directly commercialized for mine communications including the up-hole power amplifier and the data fusion engine. 
Airborne time-domain EM systems applied to mineral exploration

The first operational fixed-wing airborne electromagnetic (AEM) system was introduced in 1948 and was followed by the first operational helicopter AEM system in 1955. There has been parallel development of both fixed-wing and helicopter AEM since that time and, up to the early 1960s, all these systems were frequency-domain. Since the late 1970s, fixed-wing AEM systems have been primarily time-domain while the helicopter systems remained primarily frequency-domain. Recently, there has been development and introduction of helicopter time-domain AEM systems. The pace of introduction has been such that, today, the number of time-domain systems in use is equal to the number of frequency-domain systems. The data fusion engine developed under this research has the potential to be used commercially in this application to increase the fidelity of EM measurements made by these survey aircraft. 


\section{Conclusions}

1. The usage of data fusion methods can extend the operational capabilities of an EM telemetry system. Project test results showed that information from multiple receive antennas can be fused, in real-time, resulting in a measurable increase in a communications receiver's ability to reject ambient environmental noise. Increasing a receiver's noise rejection capability provides the receiver with the ability to recover and demodulate very small amplitude signals in the presence of large amounts of interfering ambient noise.

2. Data fusion technology can be employed to create a rugged, commercially viable telemetry system for usage within the energy exploration market. A rugged, portable prototype of a complete EM telemetry system was developed during this project. This system was designed specifically to service the energy exploration market and consists of the following components: a 16-channel surface receiver; a surface communications power amplifier; and a downhole power amplifier and receiver.

\section{The data fusion based EM telemetry system developed during this project} has direct commercialization potential.

We currently have ongoing discussions with three large energy services firms with the goal of licensing the use of portions of the system for MWD. We have segmented elements of the system bundled for use in permanent monitoring applications and are currently in negotiations to license this technology as well. We are scheduled to run a comparative test against an existing commercial EM tool in an operational well during the first quarter of 2006. 


\section{Appendix A}

\section{Sample \\ Performance Test Procedure}


Appendix A: Sample Performance Test Procedure

\section{Deep Trek Test-well Performance Test}

\section{OVERVIEW}

Two types of testing will be conducted:

Recording of downlink signal and signal plus noise waveforms at the downhole tool.

Recording of uplink signal and signal plus noise waveforms at the surface receiver.

The testing will be facilitated using the downhole test module which consists of a receiver section and a power amplifier section. The receiver hardware and firmware supports waveform logging to the on-board flash memory. A 32M-bit flash device is included which will supports up to 30 minutes of waveform logging. A downlink "RECORD" command causes the receiver to record "LENGTH" seconds of waveform at "FREQUENCY" sample rate with "GAIN" receiver front end gain applied by the receiver signal conditioning hardware.

The test module will be used in conjunction with a fiberglass gap sub used during previous EM Testing.

The overall configuration of the gap sub/antenna assembly is shown on the next page (Figure 1). The standard gap configuration consists of a fiberglass cylinder, with a 1.5 inch bore, joined to a metal I.F. box and pin.

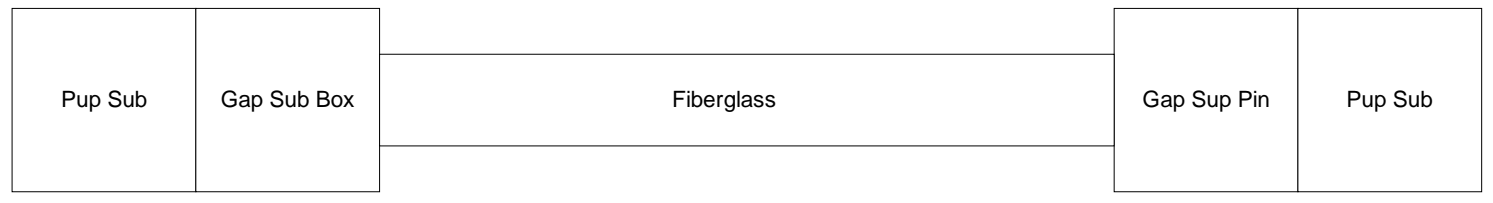

Figure 1. Gap Antenna Assembly

\section{Testing Schedule}

The following is a scheduling overview for the above noted testing.

\section{Day 1 -}




\section{Appendix A: Sample Performance Test Procedure}

Configure the test module in the well using the standard configuration gap sub. Connect the test module (+) output to the string area below the gap sub. Run-in to 2500' and record downlinks at the tool and uplinks at the surface receiver. Rotate the string at various RPM rates and record downlinks at the tool and uplinks at the surface receiver. Raise and lower the tubing string a distance of plus and minus one stand and record downlinks at the tool and uplinks at the surface receiver. Deploy the surface sensors and antennas in various configurations and record downlinks at the tool and uplinks at the surface receiver. Leave tool in well overnight.

\section{Day 2 -}

Run-in to 5000' and record downlinks at the tool and uplinks at the surface receiver. Rotate the string at various RPM rates and record downlinks at the tool and uplinks at the surface receiver. Raise and lower the tubing string a distance of plus and minus one stand and record downlinks at the tool and uplinks at the surface receiver. Deploy the surface sensors and antennas in various configurations and record downlinks at the tool and uplinks at the surface receiver. Pull tool out of well and download tool data.

\section{DETAILED TEST PROCEDURE}

\section{Well Site Layout}

The following depicts the well site layout for the tests to be conducted on the Test Well (Figures 3 and 4). 


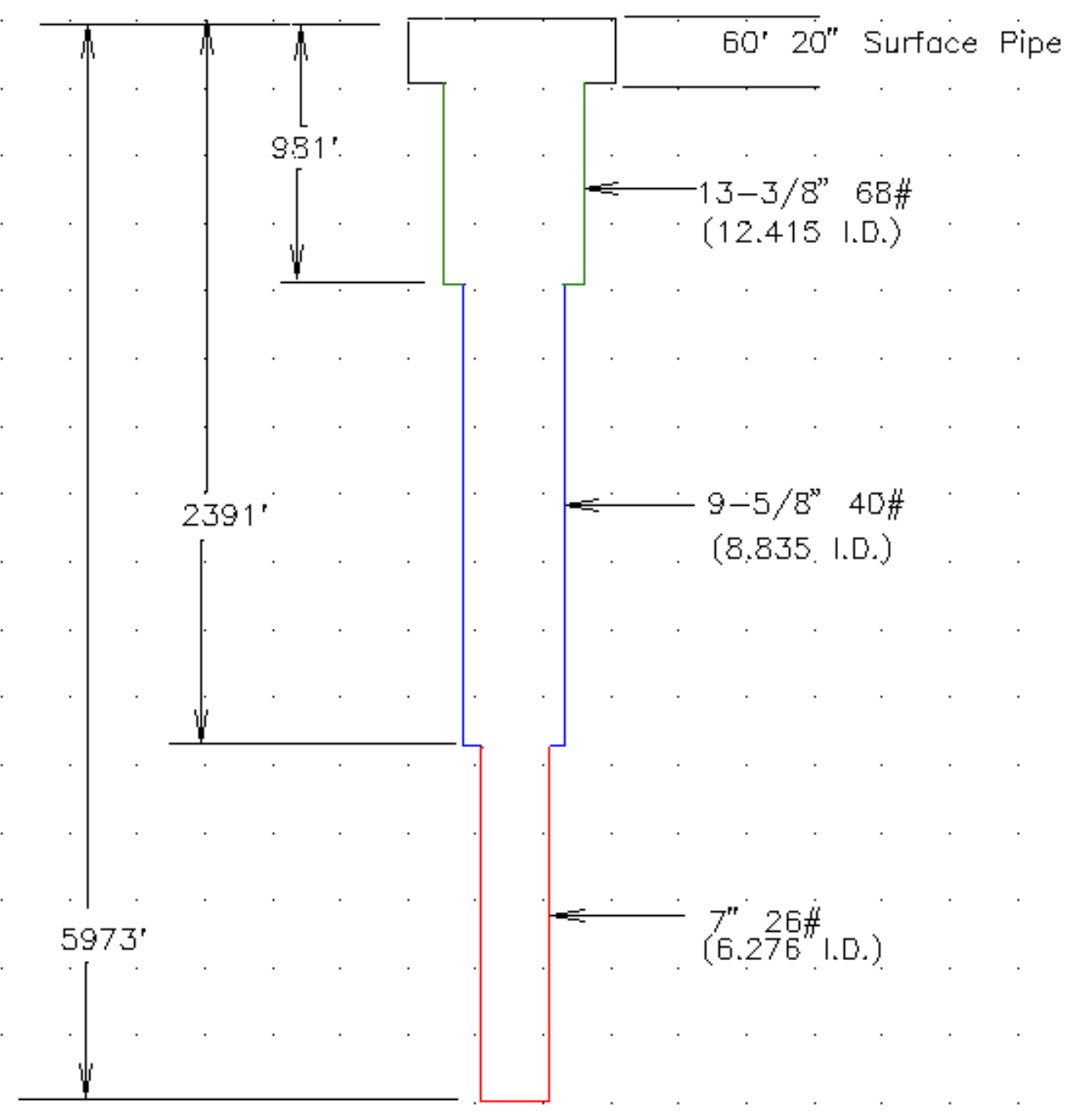

Figure 3. Test Well.

\section{Uphole Configuration}

The equipment and receiving antenna lines will be initially laid out as show below (Figure 4). During the testing it will be necessary to reposition the magnetometer, accelerometer and antenna lines to determine how these variations affect the received signal and noise. The position variations for these configurations will be documented on the data sheet. The EM Receiver and associated computer equipment will be located in the rig dog house. 
Appendix A: Sample Performance Test Procedure

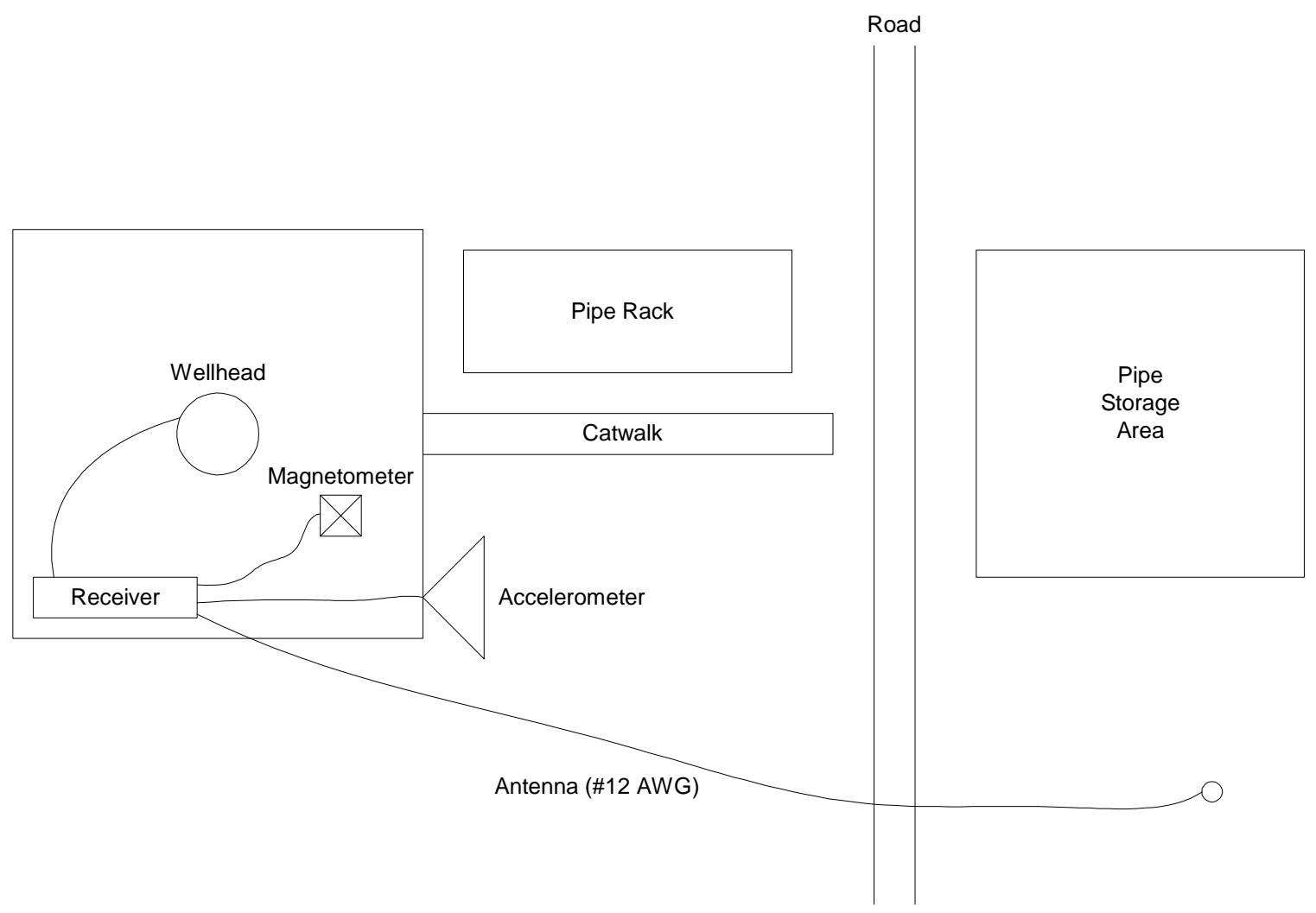

Figure 4. Well Site Initial Layout. 
Appendix A: Sample Performance Test Procedure

\section{Downhole Configuration}

The sketch below (Figure 5) depicts the configuration of the downhole tool. Place one stand of 3-1/2 EUE below the gap sub to simulate the BHA.

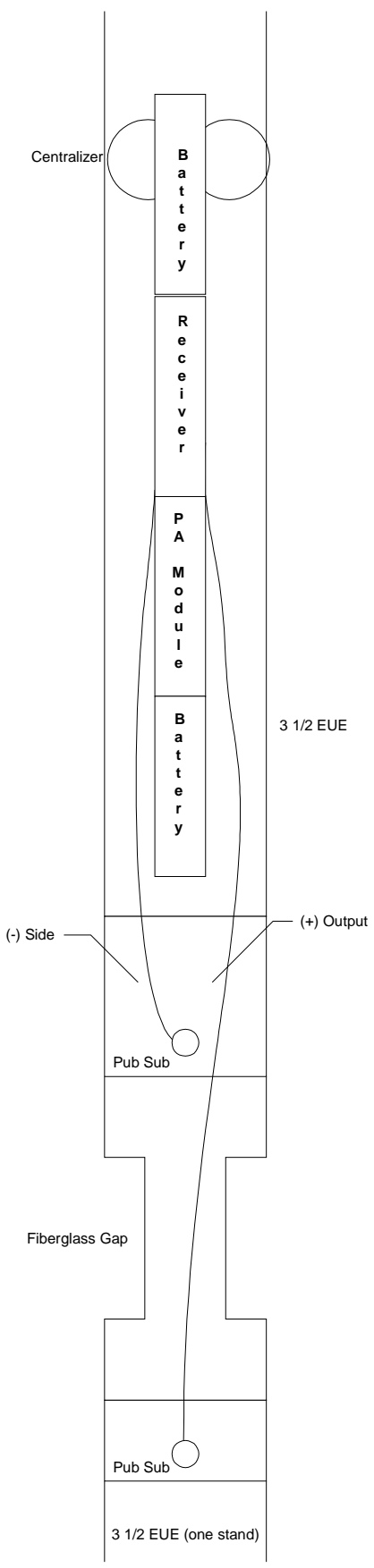

Figure 5. Downhole Tool Configuration. 